Supporting Information

\title{
Free-Standing Graphene Oxide-Chitin Nanocrystal Composite Membrane for Dye Adsorption and Oil/Water Separation
}

\author{
Xianfeng Ou, Xiaohan Yang, Jingqi Zheng, and Mingxian Liu* \\ Department of Materials Science and Engineering, Jinan University, 601 \\ Huangpu Avenue West, Tianhe District, Guangzhou 510632, China \\ *Corresponding author. Email: liumx@jnu.edu.cn
}



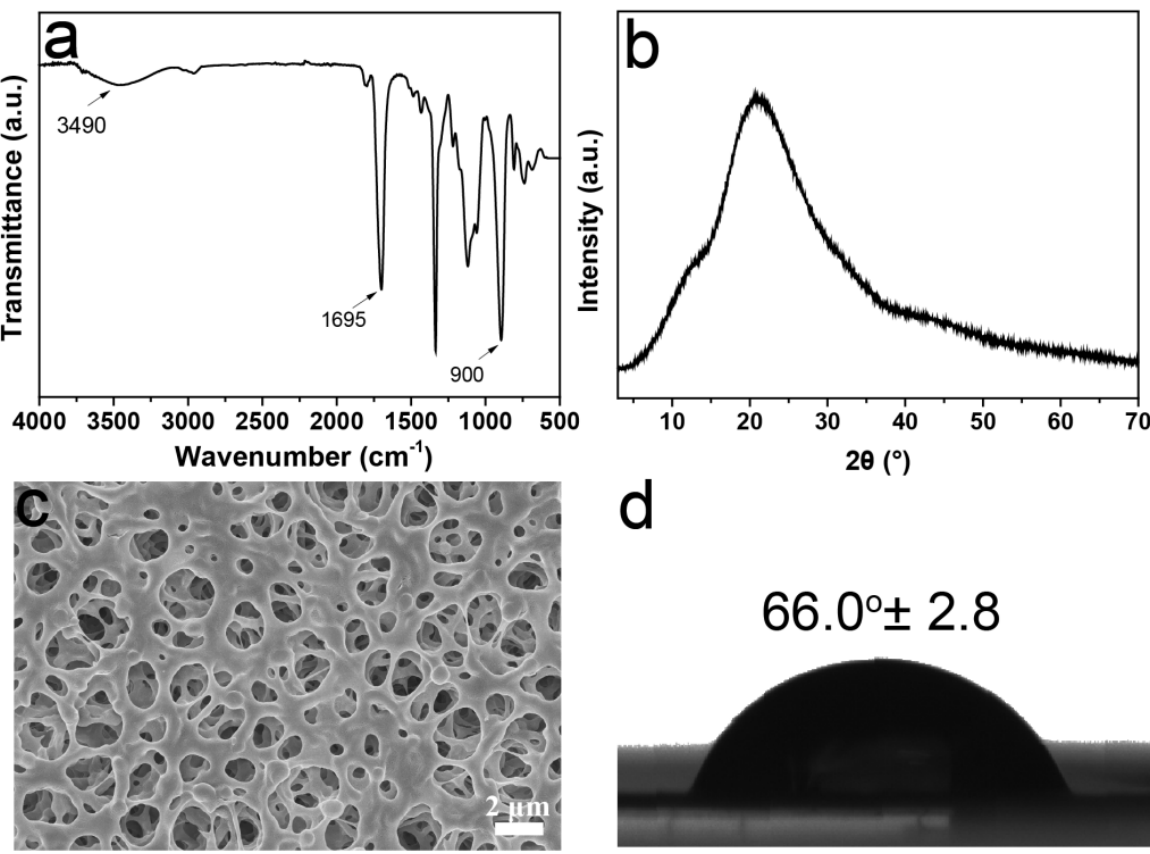

d

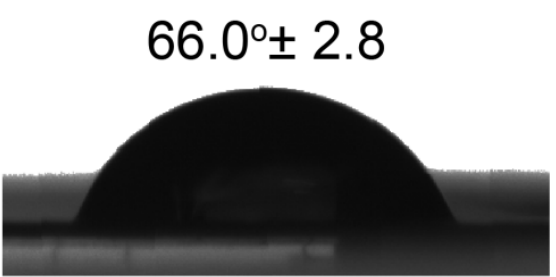

Figure S1. IR (a), XRD (b), SEM (c), water contact angle (d) of CAM.

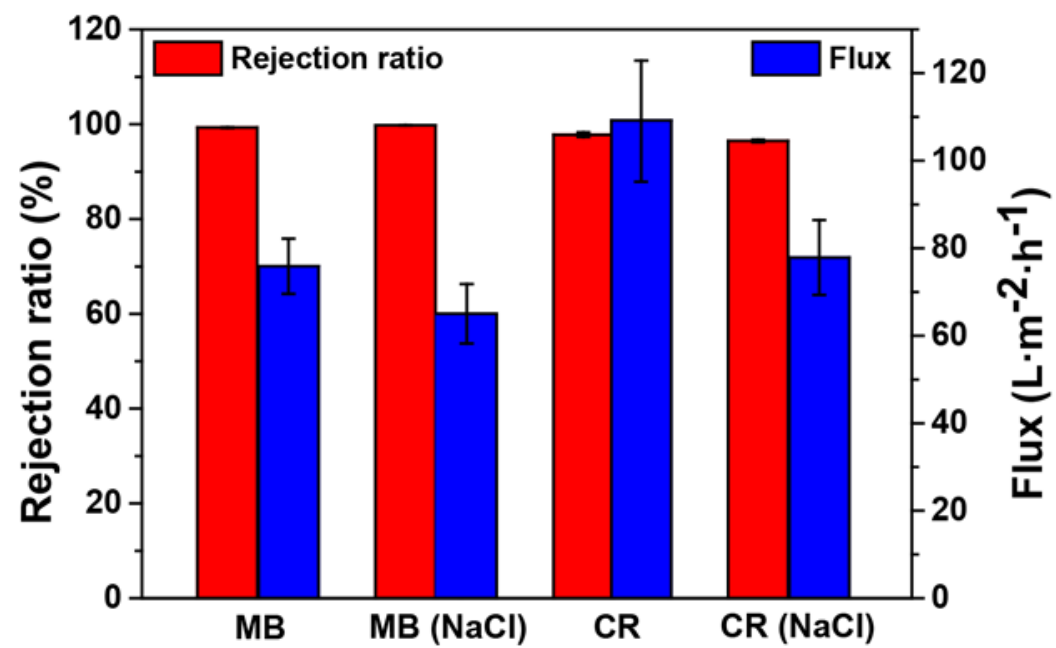

Figure S2. Rejection ratios and fluxes of pure dyes and salt containing dyes solution on M4. 